\title{
Optimization of multi-response dynamic systems using multiple regression-based weighted signal-to-noise ratio
}

\author{
Susanta Kumar Gauri ${ }^{a^{*}}$ and Surajit Pal ${ }^{\mathrm{b}}$
}

${ }^{a}$ SQC \& OR Unit, Indian Statistical Institute, 203, B. T. Road, Kolkata-700108, India

${ }^{b} S Q C \&$ \&R Unit, Indian Statistical Institute, 110, Nelson Manickam Road, Chennai- 600029, India

CHRONICLE ABSTRACT

Article history:

Received March 42016

Received in Revised Format

April 272016

Accepted May 142016

Available online

May 162016

Keywords:

Dynamic system

Multiple responses

Optimization

Composite desirability function

Multiple regression

Weighted signal-to-noise ratio

\begin{abstract}
A dynamic system differs from a static system in that it contains signal factor and the target value depends on the level of the signal factor set by the system operator. The aim of optimizing a multi-response dynamic system is to find a setting combination of input controllable factors that would result in optimum values of all response variables at all signal levels. The most commonly used performance metric for optimizing a multi-response dynamic system is the composite desirability function (CDF). The advantage of using CDF is that it is a simple unit less measure and it has a good foundation in statistical practice. However, the problem with the CDF is that it does not consider the variability of the individual response variables. Moreover, if the specification limits for the response variables are not provided the CDF cannot be computed. In this paper, a new performance metric for multi-response dynamic system, called multiple regression-based weighted signal-to-noise ratio (MRWSN) is proposed, which overcome the limitations of CDF. Two sets of experimental data on multi-response dynamic systems, taken from literature, are analysed using both CDF-based and the proposed MRWSN-based approaches for optimization. The results show that the MRWSN-based approach also results in substantially better optimization performance than the CDF-based approach.
\end{abstract}

(C) 2017 Growing Science Ltd. All rights reserved

\section{Introduction}

The usefulness of Taguchi method (Taguchi, 1986) in optimizing the parameter design in static as well as dynamic system has been well established. In a static system, a response variable representing the output characteristic of the system has a fixed target value. But in a dynamic system, the target value of a response variable depends on the level of the signal factor set by the system operator. For example, signal factor may be the steering angle in the steering mechanism of an automobile or the speed control setting of a fan. In other words, a dynamic system has multiple target values of a response variable depending on the setting of signal variable of the system. Most of the modern manufacturing processes have several response variables and the process needs to be optimized for all response variables. Extensive research works have been carried out aiming to resolve the multi-response optimization

* Corresponding author. Tel.: +91-033-2575-5951(O), +91-033-2668-0473(R), Fax.: +91-033-2577-6042

E-mail: susantagauri@hotmail.com (S. K. Gauri)

(C) 2017 Growing Science Ltd. All rights reserved. doi: $10.5267 /$ j. ijiec.2016.6.001 
problem in a static system (Derringer \& Suich, 1980; Khuri \& Conlon, 1981; Pignatiello, 1993; Su \& Tong, 1997; Tong \& Hsieh, 2000; Wu, 2004; Liao, 2006; Tong et al., 2007; Jeong \& Kim, 2009; Pal \& Gauri, 2010; Wang et al., 2016). Product/process design with a dynamic system offers the flexibility needed to satisfy customer requirements and can enhance a manufacturer's competitiveness. In recent time, therefore, many researchers (Miller \& Wu, 1996; Wasserman, 1996; McCaskey \& Tsui, 1997; Tsui, 2001; Joseph \& Wu, 2002; Chen, 2003; Lesperance \& Park, 2003; Su et al., 2005; Bae \& Tsui, 2006) have been motivated to study the robust design problem concerning the dynamic systems. However, all these research articles are focused on optimization of a single-response dynamic system.

Industry has increasingly emphasized developing procedures capable of simultaneously optimizing the multi-response dynamic systems in light of the increasing complexity of modern product design. To cope with the need of the modern industries, several studies (Tong et al., 2001; Tong et al., 2004; Hsieh et al., 2005; Wang \& Tong, 2004; Wu \& Yeh, 2005; Chang, 2006; Wang, 2007; Chang, 2008; Tong et al., 2008; Wu, 2009; Chang \& Chen, 2011; Gauri, 2014) have proposed different procedures for optimizing a multi-response dynamic system. The goal of optimizing a multi-response dynamic system is to find a setting combination of control factors (controllable variables) that would result in the optimum values of all response variables at all signal levels. Generally, it is very difficult to obtain such a combination, because optimum values of one response variable may lead to non-optimum values for the remaining response variables. Hence, it is desirable to find a best setting combination of control factor levels that would result in an optimal compromise of response variables. Here optimal compromise means each response variable is as close as possible to its target value at each signal level and with minimum variability around that target value.

Most of the researchers have attempted to optimize multi-response dynamic system using Derringer and Suich's (1980) composite desirability function (CDF) as a performance metric. Tong et al (2001), Hsieh et al (2005), and $\mathrm{Wu}(2009)$ have modelled the response variables using response surface methodology (RSM) and then determined the optimal settings of the control factors by maximizing an overall performance measure (OPI), which is essentially the CDF. On the other hand, Chang (2006), Chang (2008) and Chang and Chen (2011) used artificial neural networks (ANN) for modelling the response functions and then obtained the optimal settings of the control factors by considering OPI, which is essentially CDF, as the performance metric.

The basic advantage of using CDF as performance metric is that it is a simple unit less measure and it has a good foundation in statistical practice. However, if the specification limits for the response variables are not provided, the CDF cannot be computed. Another disadvantage with this metric is that it does not take into consideration the variability of individual response variables. Hence, a CDF-based approach may produce an optimal solution where the expected means of the response variables at a signal level is very close to their target values, but variability of one or more response variables around the target is very high, which may not be acceptable by the engineers.

Pal and Gauri (2010) have shown that many limitations of the CDF-based approach, encountered during optimization of a multi-response static system, can be overcome by using multiple regression-based weighted signal-to-noise ratio (MRWSN) as the performance metric. The advantages of MRWSN as a performance measure are that (a) signal-to-noise ( $\mathrm{SN}$ ) ratio for a response variable can be computed even when the specification limits and target values for the response variable are unknown, (b) SN ratio takes care of both location (mean) and dispersion (variability) of a response variable and (c) since SN ratios are always expressed in decibels $(\mathrm{dB})$ whatever be the units of measurements of the individual responses, there is no problem in summing the $\mathrm{SN}$ ratios of the individual responses.

The aim of the current research is to develop an appropriate procedure for optimizing the multi-response dynamic systems using MRWSN as the performance metric and evaluate the effectiveness of the MRWSN-based optimization approach. The article is organized as follows: the second section outlines 
briefly about the dynamic systems and reported various approaches for its optimization. The third section describes formulation of commonly used performance metric, called CDF, for the multi-response dynamic system. The formulation of the proposed performance metric, called MRWSN, for the multiresponse dynamic system is presented in the fourth section. The procedure for implementation of the MRWSN-based optimization approach is described in section five. In the sixth section, analysis of two experimental datasets taken from literature and related results are presented. We conclude in the final section.

\section{Dynamic Systems and its Optimization}

Dynamic systems are those where the response variable does not have a fixed target value and the target value of the response variable depends on the level of a control factor (called signal factor) set by the system operator. For example, the steering mechanism of an automobile or speed controller of a fan is a dynamic system. In the case of an automobile steering system, the signal may be the angle of the steering wheel and the response may be the direction of motion or turning radius of the car. In a dynamic system, a response is expected to assume different target values for different levels of the signal factor and so it is often called multi-target system (Joseph \& Wu, 2002). In case of a dynamic system, the signal-response relationship is of prime importance and therefore, it is also known as signal-response system (Miller \& $\mathrm{Wu}, 1996)$. A single-response dynamic system contains only one response variable. On the other hand, a multi-response dynamic system contains more than one response variables and responses are expected to assume different target values as a result of changes in the levels of the signal factor.

\subsection{Taguchi method and related works for optimizing a single-response dynamic system}

Taguchi (1986) first took interest in designing robust dynamic systems and he considered only the singleresponse dynamic systems. For a dynamic system, according to Taguchi (1986), ideal quality is based on the ideal relationship between the signal factor and the response variable, and quality loss is caused by deviations from the ideal relationship. So, significant quality improvement can be achieved by first defining a system's ideal function and then using designed experiments to search for an optimal design which minimizes deviations from this ideal function. Taguchi (1986) assumed that a linear relationship exists between the response variable $(Y)$ and the signal factor $(M)$ of the system, and thus the ideal function can be expressed as follows:

$$
Y=\beta M+\varepsilon,
$$

where $\beta$ is the slope or system sensitivity, and $\varepsilon$ denotes the random error. Here $\varepsilon$ is assumed to follow a normal distribution with a mean of zero and variance of $\sigma_{\beta}^{2}$. The deviation from the ideal function is represented by the variability of the dynamic system, i.e. $\sigma_{\beta}^{2}$. The objective is to determine the best combination of input controllable variables so that the system achieves the respective target value at each level of the signal factor and with minimum variability around the target value. For the purpose of optimization of a single-response dynamic system which has one response variable $(Y)$ whose value are determined by $p$ controllable variables $\mathbf{X}=\left(X_{1}, X_{2}, \ldots, X_{p}\right)$, a signal factor $(M)$ and a noise factor $(Z)$, Taguchi (1986) proposes the following guidelines for designing the experimental plan. Depending on the number of controllable factors (variables) and their levels, select first the most appropriate inner orthogonal array and accordingly determine various trial conditions or experimental runs. On the other hand, determine the noise factor and signal factor levels under which samples are to be tested. Then, conduct the experiments in such a way that different samples under each trial condition are exposed to different combinations of noise factor and signal factor levels. Let $y_{j k l}(j=1,2, \ldots, s ; l=1,2, \ldots, n)$ are the observed values of the response variable at the combination of $j^{\text {th }}$ level of signal factor $\left(M_{j}\right)$ and $l^{\text {th }}$ 
level of noise factor $\left(Z_{l}\right)$ under $k^{\text {th }}$ trial condition i.e. vector of control factors levels $\mathbf{x}_{k}=\left(x_{1}, x_{2}, \ldots, x_{p}\right)$. Then, according to Taguchi (1986), the slope $\left(\beta_{k}\right)$ and the variability around the slope $\left(\sigma_{\beta_{k}}^{2}\right)$ under the $k^{\text {th }}$ trial condition can be obtained using the following equations respectively:

$$
\begin{aligned}
& \beta_{k}=\frac{\sum_{j=1 l=1}^{s} \sum^{n}\left(y_{j k l} M_{j}\right)}{\sum_{j=1}^{s} M_{j}^{2}} \\
& \sigma_{\beta_{k}}^{2}=\frac{1}{s n-1} \sum_{j=1 l=1}^{s} \sum_{j}^{n}\left(y_{j k l}-\beta_{k} M_{j}\right)^{2}
\end{aligned}
$$

The aim of robust design is to find the combination of controllable factors so that the effect of noise factors on the target response of the dynamic system is as small as possible. Taguchi, therefore, uses SN ratio to judge the performance under an experimental run or trial condition. For a dynamic system, the $\mathrm{SN}$ ratio under $k^{\text {th }}$ experimental run is estimated as follows:

$$
\mathrm{SNR}_{k}=10 \log _{10}\left(\frac{\beta_{k}^{2}}{\sigma_{\beta_{k}}^{2}}\right)
$$

The larger SN ratio means the response has less deviation from its target. It may be noted that the target value of the slope $(\beta)$ is different for different type of response variables. For dynamic nominal-the-best (DNTB), dynamic larger-the-better (DLTB), and dynamic smaller-the-better (DSTB) type response variables, the desired value of slopes are $0<\beta<\infty, \beta=\infty$, and $\beta=0$ respectively. In analysing the experimental data, Taguchi (1986) proposed a two-step procedure. In the first step, the settings of the controllable variables $X_{1}, X_{2}, \ldots, X_{p}$ are determined in such a way that $\mathrm{SN}$ ratio is maximized and in the second step, the slope $(\beta)$ is adjusted by a suitable scaling factor to the desired slope. Any control factor that has a large effect on $\beta$ but no effect on the variability $\left(\sigma_{\beta}^{2}\right)$ is considered as a scaling factor. Miller and $\mathrm{Wu}$ (1996) observed that the conventional Taguchi approach for modelling the ideal relationship of a dynamic system lacks a solid basis. They proposed two strategies for modelling a dynamic system and analysing data. These are (i) performance measure modelling (PMM), which is termed as loss model (LM) by Tsui (1999) and (ii) response function modelling (RFM). In PMM approach, each performance measure $\left(\beta\right.$ and $\sigma_{\beta}^{2}$ ) is modelled as a function of control factors ( $\mathbf{X}$ ) and in RFM approach, performance measures is modelled as a function of the control factors $(\mathbf{X})$ and signal factor $(M)$. The advantage of the RFM approach is that it can reveal how specific control factor interact with specific noise factor. Tsui (1999) proposes a response modelling (RM) approach which directly models the response as a function of the control, noise and signal factors. Tsui (2001) investigated the performances of RM, PMM/LM and RFM approaches for optimizing a single-response dynamic system and concluded that the RM approach has more potential to reach to an optimal solution. Joseph and $\mathrm{Wu}$ (2002) have formulated the robust parameter design of dynamic system as a mathematical programming problem. Lesperance and Park (2003) have suggested to use a joint generalized linear model (GLM) so that model assumptions can be investigated using residual analysis. Chen (2003) has proposed a stochastic optimization modelling procedure for optimizing a single response dynamic system. Bae and Tsui (2006) have observed that the GLM-RM approach can provide more reliable results.

\subsection{The approaches for optimizing a multi-response dynamic system}

All the above research articles are focussed on optimization of a single-response dynamic system. Realizing the need of the modern industries, some authors took research interest in developing 
appropriate procedure for optimizing multi-response dynamic systems. Different researchers have advocated different approaches for modelling the multiple responses but most of them (Tong et al., 2001; Hsieh et al., 2004; Chang, 2006; Chang, 2008; Wu, 2009; Chang and Chen, 2011) have used CDF as the performance metric for optimization of the multi-response dynamic systems. Tong et al. (2004), Wang and Tong (2004), Wu and Yeh (2005), Wang (2007) and Gauri (2014) have used different performance metrics. While overall relative closeness to the ideal solution is considered as the performance metric by Tong et al. (2004), overall grey relational grade is considered as the performance metric by Wang and Tong (2004) and Wang (2007). Wu and Yeh (2005) have derived total quality loss and minimized it to determine the optimal settings for a multi-response dynamic system, and Gauri (2014) considered overall utility value as the objective function for optimization of multi-response dynamic systems. Among the various types of performance metrics for the multi-response dynamic system, the CDF is most popular among the researchers because it is a simple unit less measure and it has a good foundation in statistical practice.

\section{Formulation of CDF for Multi-response Dynamic System}

Suppose that in a multi-response dynamic system, there are $r$ output responses $\left(Y_{1}, Y_{2}, \ldots, Y_{r}\right), s$ signal factor settings $\left(M_{1}, M_{2}, \ldots, M_{s}\right), p$ control factors $\left(X_{1}, X_{2}, \ldots, X_{p}\right)$, and $n$ noise factor settings $\left(Z_{1}, Z_{2}, \ldots, Z_{n}\right)$

. For analysing a dynamic system, the first requirement is to model the response variables appropriately. As reported by Tsui (2001), among the three approaches (RM, PMM, and RFM) for modelling the responses in a dynamic system, the RM approach has more potential to reach to an optimal solution. Most of the authors (Tong et al., 2001; Hsieh et al., 2005; Chang, 2008; Wu, 2009) using CDF as the performance metric, have also used RM approach for modelling the observed responses. Therefore, formulation of CDF is discussed here considering that RM approach is used for modelling the observed responses. Using RM approach, the observed responses in the multi-response dynamic system can be modelled as follows:

$$
y_{i j k l}=f_{i}\left(M_{j}, \mathbf{X}_{k}, Z_{l}\right)+\varepsilon_{i j k l},
$$

where $f_{i}\left(M_{j}, \mathbf{X}_{k}, Z_{l}\right)$ denotes the response function between the $i^{\text {th }}$ response and the corresponding setting at the $j^{\text {th }}$ level of signal factor $\left(M_{j}\right)$ and the $l^{\text {th }}$ level of noise factor $\left(Z_{l}\right)$ under $k^{\text {th }}$ vector of control factors levels $\mathbf{x}_{k}=\left(x_{1}, x_{2}, \ldots, x_{p}\right)$. The terms $y_{i j k l}$ and $\varepsilon_{i j k l}$ represent the values of $i^{t h}$ response and error respectively at the $j^{\text {th }}$ level of signal factor and $l^{\text {th }}$ level of noise factor under $k^{\text {th }}$ vector of control factors levels. The error is assumed to follow a normal distribution with mean value as zero and a constant variance $\sigma_{\varepsilon}^{2}$. For each response, it is assumed that a linear form exists between the response and the signal factor as shown in Eq. (1). The exponential desirability function approach to evaluate the quality of a response was introduced by Harrington (1965). The exponential desirability functions normalize an estimated response $\hat{y}$ according to the system's desire and then use exponential functions to transform the normalized value to a scale-free value $d$, called desirability. It is a value between 0 and 1 , and increases as the desirability of the response increases. Derringer and Suich (1980) presented an alternative form of desirability functions which are more flexible in the sense that these can assume a variety of shapes and they defined the CDF of $m$ responses as the geometric mean of the individual desirability. Tong et al. (2001) and Hsieh et al. (2005) have used the same CDF as the performance measure for optimizing the multi-response dynamic systems. The lack of considering the correlation between quality characteristics is a disadvantage of Derringer and Suich's (1980) desirability function. Wu and Hamada (2000), therefore, defined different desirability functions, called double-exponential desirability functions. Chang (2008) and $\mathrm{Wu}(2009)$ applied double-exponential desirability functions for optimization of multiresponse dynamic systems. 
The double-exponential desirability functions for the responses in dynamic system can be better understood by defining first the double-exponential desirability functions for the NTB, LTB and STB type responses of a static system. According to Chang (2008), the double-exponential desirability function for the NTB, LTB and STB type responses under $k^{\text {th }}$ trial condition in a static system can be formulated using the following equations, respectively.

$$
\begin{aligned}
& d_{k}^{N T B}=\exp \left(-\left|\frac{2 \hat{y}_{k}-(U S L+L S L)}{U S L-L S L}\right|\right), \\
& d_{k}^{L T B}=\exp \left(-\exp \left(-\frac{\hat{y}_{k}-L S L}{L S L}\right)\right), \\
& d_{k}^{S T B}=\exp \left(-\left(1+\frac{\hat{y}_{k}-U S L}{U S L}\right)\right),
\end{aligned}
$$

where, $\hat{y}_{k}$ and $d_{k}$ represent estimate and desirability of the response variable under $k^{\text {th }}$ trial condition, and USL and LSL stand for upper and lower specification limits respectively. A dynamic system can be regarded as a system having multiple static targets which vary depending on their signal values (Joseph $\& \mathrm{Wu}, 2002)$. Thus, for a dynamic response we can average the normalized values of all estimated responses in a specific experimental run to evaluate that run's desirability. Accordingly, Chang (2008) estimated the double-exponential desirability functions for DNTB, DLTB and DSTB type responses under $k^{\text {th }}$ trial condition in a multi-response dynamic system using the following equations respectively:

$$
\begin{aligned}
& d_{i k}^{D N T B}=\exp \left(-\frac{1}{S n} \sum_{j=1 l=1}^{s} \sum_{1=1}^{n} \mid \frac{2 \hat{y}_{i j k l}-\left(U S L_{i j}+L S L_{i j}\right)}{U S L_{i j}-L S L_{i j}}\right), \\
& d_{i k}^{D L T B}=\exp \left(-\exp \left(-\frac{1}{S n} \sum_{j=1 l=1}^{s} \frac{\hat{y}_{i j k l}-L S L_{i j}}{L S L_{i j}}\right)\right), \\
& d_{i k}^{D S T B}=\exp \left(-\left(1+\frac{1}{s n} \sum_{j=1 l=1}^{s} \sum^{n} \frac{\hat{y}_{i j k l}-U S L_{i j}}{U S L_{i j}}\right)\right),
\end{aligned}
$$

where $\hat{y}_{i j k l}$ denotes the estimate of $i^{\text {th }}$ response at $j^{\text {th }}$ level of signal factor and $l^{\text {th }}$ level of noise factor under $k^{\text {th }}$ vector of control factors levels, and $U S L_{i j}$ and $L S L_{i j}$ denotes the upper and lower specification limits for $i^{\text {th }}$ response at $j^{\text {th }}$ signal level respectively. Then, Chang (2008) evaluated the overall performance index (OPI) of the multi-response dynamic system having $r$ response variables under the $k^{\text {th }}$ vector of control factors levels as follows:

$$
\mathrm{OPI}_{k}=\left(\prod_{i=1}^{r} d_{i k}\right)^{1 / r}
$$

This OPI is essentially the CDF. In the CDF-based optimization approach for multi-response dynamic system, settings of the control factors that maximizes the OPI value is considered as the optimal settings.

\section{Formulation of the Proposed Performance Metric for Multi-response Dynamic System}

The proposed performance metric, MRWSN, is derived by integrating multiple regression technique and Taguchi's SN ratio concept. According to the conventional Taguchi method (1986), the SN ratios of the response variables for a specific experimental run in a dynamic system are obtained based on the observed values of the response variables in the experimental run under all possible signal $\times$ noise 
combinations. In the proposed method, it is suggested to predict first the values of the response variables for the experimental run under all possible signal $\times$ noise combinations based on the appropriately fitted multiple regression equations and then to compute the $\mathrm{SN}$ ratios for different response variables based on their predicted values instead of their observed values. It may be important to note that error $(\varepsilon)$ in the response model represents a random error term which is assumed to follow a normal distribution with mean value as zero and a constant variance $\sigma_{\varepsilon}^{2}$ (Tsui, 1999). However, in general, it is expected that random error will have increased variability at higher signal levels instead of having constant variability at all signal levels. Lesperance and Park (2003), therefore, recommend that fitting such a model requires the use of GLM fitting techniques with a log-link or appropriate link function. Fitting a GLM with loglink function to the experimental data would be very difficult for many quality practitioners. For simplification, we suggest to use a logarithmic transformation of the response variables and then applying multiple regression techniques for fitting the response models. The fitted models, then, will partly ensure that the variability due to random error is more at higher signal levels. Suppose, each of the $r$ response variables is related to $p$ controllable variables $\mathbf{X}=\left(X_{1}, X_{2}, \ldots, X_{p}\right)$, signal factor $(M)$ and noise factor $(N)$ by

$$
\log _{10}\left(Y_{i}\right)=b_{0}+\sum_{u=1}^{p} b_{u} X_{u}+\sum_{u, v} b_{u v} X_{u} X_{v}+M\left(\gamma_{0}+\sum_{u=1}^{p} \gamma_{u} X_{u}\right)+N\left(\delta_{0}+\sum_{u=1}^{p} \delta_{u} X_{u}\right)+\varepsilon,
$$

where $b_{u}, b_{u v}, \gamma_{u}, \delta_{u}(u=1,2, \ldots, p ; v=1,2,3, \ldots, p)$ are regression coefficients, $X_{u} \quad(u=0,1,2, \ldots, p)$ are controllable variables and $Y_{i}(i=1,2, \ldots, r)$ are $i^{\text {th }}$ response variable. It may be noted that apart from the main factors, the quadratic terms of main factors may be included in the model when there is three or more number of levels in any control and signal factors. The interaction terms of controllable variables may also be included in the model. But, it is very important to include a few control $\times$ signal and control $\times$ noise interaction terms into the response model. If a few control $\times$ signal and control $\times$ noise interaction terms are not included in the response model, $\log _{10}\left(Y_{i}\right)$ will be additive at different signal levels independent of combinations of controllable factors $\mathbf{X}$, which implies that intercept only will change but slopes of the controllable variables $\mathbf{X}$ will remain constant. As a result, it will not be possible to obtain desired predicted values of the response variables $Y_{i}(i=1,2, \ldots, r)$ at different signal levels. Let the combination of the control factors levels is $\mathbf{x}_{k}=\left(x_{1}, x_{2}, \ldots, x_{p}\right)$ under $k^{\text {th }}$ experimental run or trial condition. For the $k^{\text {th }}$ vector of control factors levels, the values of all the response variables at all possible combinations of signal $\times$ noise levels can be predicted using the fitted response models. Suppose, $\hat{y}_{i j k l}$ is the predicted value of $i^{t h}$ response variable at $j^{\text {th }}$ level of signal factor and $l^{\text {th }}$ level of noise factor under $k^{\text {th }}$ vector of control factors levels. Then, the slope $\left(\beta_{i k}\right)$ and variance around slope $\left(\sigma_{\beta_{i k}}^{2}\right)$ of the $i^{\text {th }}$ response variable under the $k^{\text {th }}$ experimental run can be estimated as follows:

$$
\begin{aligned}
& \hat{\beta}_{i k}=\frac{\sum_{j=1 l=1}^{s} \sum_{i j k l}^{n} \hat{y}_{i j k} M_{j}}{\sum_{j=1}^{s} M_{j}^{2}} \\
& \hat{\sigma}_{\beta_{i k}}^{2}=\frac{1}{s n-1} \sum_{j=1 l=1}^{s} \sum_{l}^{n}\left(\hat{y}_{i j k l}-\hat{\beta}_{i} M_{j}\right)^{2}
\end{aligned}
$$

Therefore, the SN ratio of the $i^{\text {th }}$ response variable in the experimental run can be obtained using Eq. (16) shown below. It is important to observe that the forms of Eq. (4) and Eq. (16) are the same. The two equations differ only with respect to usage of observed values or predicted values for making the required computations. In Eq. (4), $\beta$ and $\sigma_{\beta}^{2}$ are calculated based on the observed values of a response variable under a trial condition. On the other hand, in Eq. (16), $\hat{\beta}$ and $\hat{\sigma}_{\beta}^{2}$ are calculated using the predicted values 
of a response variable based on the fitted multiple regression equation, under a trial condition. Therefore, the computed SN ratio that is obtained using Eq. (16) is called as multiple regression-based signal-tonoise ratio (MRSN). Eq. (16) gives estimate of the MRSN value of the $i^{\text {th }}$ response variable under $k^{\text {th }}$ trial condition or vector of control factors levels.

$$
\operatorname{MRSN}_{i k}=10 \log _{10}\left(\frac{\hat{\beta}_{i k}^{2}}{\hat{\sigma}_{\beta_{i k}}^{2}}\right) \text {. }
$$

Likewise SN ratio, MRSN is always expressed in decibel (dB) unit and higher MRSN implies better quality. For a multi-response dynamic system, MRWSN can be taken as the overall performance measure. The MRWSN of the multi-response dynamic system under $k^{\text {th }}$ trial condition can be obtained using the following equation:

$$
\operatorname{MRWSN}_{k}=\sum_{i=1}^{r} w_{i} \times \operatorname{MRSN}_{i k}=\sum_{i=1}^{r} w_{i} \times\left[10 \log _{10}\left(\frac{\hat{\beta}_{i k}^{2}}{\hat{\sigma}_{\beta_{i k}}^{2}}\right)\right] \text {, }
$$

where, $\mathrm{MRSN}_{i k}$ is the multiple regression-based SN ratio of the $i^{\text {th }}$ response variable under $k^{\text {th }}$ trial condition, $w_{i}$ is the relative weight of the $i^{t h}$ response variable, and $\sum_{i=1}^{r} w_{i}=1$. It is suggested to consider $w_{i}=1 / r$, if the relative importance of the response variables are unknown. It may be noted that MRWSN is essentially a function of the input controllable variables or control factors. Since higher MRSN implies better quality, it is desired that the process conditions are set in such a way that it result in the maximum MRWSN value. It is worth to mention that MRWSN as a performance measure has the following advantages over the CDF: (1) MRWSN can be computed even when the specification limits and target values for one or more response variables are not known and (2) MRWSN take into consideration the variability of the response variables.

\section{The Procedure for Implementation of the Proposed Optimization Approach}

A multi-response dynamic system can be optimized using MRWSN as the performance metric in the following seven steps:

Step 1: Design the experimental plan, carry out the experimentation and record the experimental observations.

Step 2: Establish the most appropriate multiple regression equations (response models) for prediction of the response variables based on the values of the control factors, signal factor and noise factor levels.

For fitting the multiple regression equations for prediction of a response variable, it is suggested to consider the log transformed values of the response variable as the dependent variable to partly ensure that the variability due to random error is more at higher signal levels. The option of performing multiple linear regression analysis is available in Microsoft Excel as well as in many statistical software packages, e.g. MINITAB, STATISTICA, SPSS etc. The regression coefficients $R^{2}$ and adjusted $R^{2}$ are considered to drop unnecessary terms from the model and to include only those terms that have some contributions on the dependent variable. Diagnostic checks for validating the regression models must be performed. Using ANOVA and F-test for significance of regression, the adequacy of model can be checked. A residual analysis in terms of various plots, e.g. normality plot of residuals, plot of residual versus predicted values and plot of residual versus individual regression variable etc. should be examined to detect possible anomalies. If, after the diagnostic checks, no serious violations of model assumptions are detected, then the regression equation can be assumed to be adequate fit to predict the dependent variable. More details about fitting of multiple regression equations and the diagnostic checks are available in Montgomery et al. (2012). 
Step 3: Choose an arbitrary setting combination of control factors levels (say, the existing combination) $\mathbf{x}_{k}$, and compute the predicted values of all response variables under the setting combination using respective regression equations for all possible signal $\times$ noise levels.

Step 4: Compute slope $\left(\hat{\beta}_{i k}\right)$, variance around the slope $\left(\hat{\sigma}_{\beta_{i k}}^{2}\right)$ and $\operatorname{MRSN}_{i k}(i=1,2, \ldots, r)$ under the vector of control factors levels $\mathbf{x}_{k}$ using Eqs. (14-16), respectively.

Step 5: Compute MRWSN $_{k}$ under the vector of control factors levels $\mathbf{x}_{k}$ using Eq. (17).

It may be noted that MRWSN $_{k}$ is essentially a function of the input controllable variables or control factors. Here the aim is to determine the level values of the input controllable variables that will maximize the MRWSN $_{k}$ value, which can be determined by changing level values of the input controllable variables and comparing the resulting MRWSN $_{k}$ values. It has been observed that if all the computations are carried out in Excel worksheet, this enumerative search for finding the optimal level values of the control factors can be performed very effectively using the 'Solver' tool of Microsoft Excel package. It is a kind of 'what if' analysis that finds the optimal value of a target cell by changing values in cells used to calculate the target cell. The 'Solver' tool employs the generalized reduced gradient (GRG) method for optimization, proposed by Del Castillo and Montgomery (1993). Examples on usage of 'Solver' tool is available in Pal and Gauri (2010).

Step 6: Determine the level values of the input controllable variables that maximize the $\mathrm{MRWSN}_{k}$ value, using 'Solver' tool of Microsoft Excel package.

While running the 'Solver' tool it is necessary to specify the range of levels for the input variables. In certain cases, where one or more input variables take only discrete values, the integer restriction (IR) for those input variables need to be specified. Sometimes, one may have to add an additional constraint to keep the slope of the DNTB variable closer to its target slope value. Unless technically there is IR for a response variable, ideally, one should not restrict the optimization to only the actual experimental design settings because that may lead to a suboptimal solution. Because the MRWSN metric is defined based on regression models, the proposed method can provide an optimal solution over the entire experimental region of the input controllable variables while using the 'Solver' tool of Microsoft Excel package.

Step 7: Obtain the expected value of each response variable at each signal level at the derived optimal condition using the relevant multiple regression equation. Then carry out the confirmatory trial and verify that the actual results conform to the expected results.

\section{Evaluation of Optimization Performance of the Proposed Method}

Under the current research, there is no scope for collection of primary data from industry. Therefore, it is decided to analyse two sets of secondary data, i.e. published data in the literature as two separate case studies and compute the values of some appropriately defined utility measures for evaluation of the performance of the proposed MRWSN-based optimization method.

\subsection{Utility measures for comparing optimization performance}

From a process engineer's perspective, the best solution should result in the minimum total quality loss implying that maximum total SN ratio (TSN) according to Taguchi philosophy (1986). Therefore, it is decided that the expected TSN at the derived optimal process condition will be considered as an important utility metric for comparison of performance of CDF and MRWSN-based optimization methods. But many statisticians (Leon et al., 1987; Box, 1988) criticised Taguchi's SN ratio concept. Lin and Tu's (1995) mean square errors (MSE) function has a very good foundation in statistical practice. The MSE 
of $i^{\text {th }}$ response variable at $j^{\text {th }}$ level of signal factor under $k^{\text {th }}$ vector of control factors levels can be computed using the following equation:

$$
\operatorname{MSE}_{i j k}=\frac{1}{n} \sum_{l=1}^{n}\left(\hat{y}_{i j k l}-T_{i j}\right)^{2}
$$

where $\hat{y}_{i j k l}$ is the predicted value of $i^{\text {th }}$ response variable at $j^{\text {th }}$ level of signal factor and $l^{\text {th }}$ level of noise factor under $k^{\text {th }}$ vector of control factors levels and $T_{i j}$ is the target value of $i^{\text {th }}$ response variable at $j^{\text {th }}$ level of signal factor. For a response variable in a dynamic system, the target values of the response variable change according to the signal factor levels. So, PMSE of $i^{\text {th }}$ response variable under $k^{\text {th }}$ vector of control factors levels can be computed using the following equation:

$$
\operatorname{PMSE}_{i k}=\frac{1}{s n} \sum_{j=1 l=1}^{s} \sum_{l=1}^{n}\left(\hat{y}_{i j k l}-T_{i j}\right)^{2} \text {. }
$$

are very close to its target values with reasonably low variability at all the signal levels, the PMSE $_{i k}$ will be quite small. It is important to note that for computation of $\mathrm{PMSE}_{i k}$ value, it is necessary to know the target values of $i^{\text {th }}$ response variable at all signal levels $(j=1,2, \ldots, s)$. However, often the target values are not specified for the DSTB and DLTB type responses variables. Therefore, PMSE $_{i k}$ value cannot be computed straightway for these response variables. It is decided to make the following assumptions about the target values of DSTB and DLTB type variables at different signal levels to facilitate computations of $\mathrm{PMSE}_{i k}$ value for these variables. It can be found from Eq. (1) that the value of a response variable is equal to the slope $\beta$ when $M=1$, i.e. at the signal level $M_{1}$. Therefore, it is decided to compute the $\beta$ values from the experimental observations at signal level $M_{1}$ in all the experimental runs, and then to consider the smallest $\beta$ values among all the experimental runs as the target value of the DSTB variable at signal level $M_{1}$, and its multiples as the target values of the DSTB variable at signal levels $M_{2}$ and $M_{3}$ respectively. Similarly, the largest $\beta$ value, computed from the experimental observations at signal level $M_{1}$ is considered as the target value of the DLTB variable at signal level $M_{1}$, and its multiples are considered as the target values of the DLTB variable at signal levels $M_{2}$ and $M_{3}$, respectively. The multivariate PMSE function (MPMSE) under an experimental run, which may be obtained by pooling the PMSE functions of the individual response variables under the experimental run, can be the most appropriate overall utility measure. But the problem with the MPMSE function is that it will be quite difficult to explain its unit because the PMSE for different response variables may have different units of measurements. Therefore, the PMSE values of the individual response variables are considered as another utility measure for comparison of optimization performance instead of MPMSE value.

\subsection{The experimental data}

Chang (2008) illustrated application of his proposed data mining approach for optimizing multi-response dynamic systems using a case study adopted from Chang (2006). This case involves simultaneous optimization of three dynamic response variables named $Y_{1}, Y_{2}$ and $Y_{3}$. Among these, $Y_{1}$ is DLTB type, $Y_{2}$ is DNTB type and $Y_{3}$ is DSTB type variable. In this case, six control factors A - F, each at three levels $(1,2$, and 3$)$, were considered and arranged in a standard $L_{18}$ orthogonal array as shown in Table 1 . The signal factor of the case had three levels named $M_{1}, M_{2}$ and $M_{3}$, the corresponding values of which were 10, 20 and 30, respectively. Two levels $\left(N_{1}\right.$ and $\left.N_{2}\right)$ of noise factor were considered in this case. The specifications for the response variables and the experimental data of Chang (2006) are reproduced in Table 2 and Table 3 respectively. The experimental data of Chang (2006) are analysed as case study 1. 
Table 1

Experimental design used by Chang (2006)

\begin{tabular}{|c|c|c|c|c|c|c|}
\hline \multirow{2}{*}{ Trial no. } & \multicolumn{6}{|c|}{ Factors and levels } \\
\hline & A & B & $\mathrm{C}$ & $\mathrm{D}$ & $\mathrm{E}$ & $\mathrm{F}$ \\
\hline & 1 & 1 & 1 & 1 & 1 & 1 \\
\hline 2 & 2 & 2 & 2 & 2 & 2 & 2 \\
\hline 3 & 3 & 3 & 3 & 3 & 3 & 3 \\
\hline 4 & 1 & 1 & 2 & 2 & 3 & 3 \\
\hline 5 & 2 & 2 & 3 & 3 & 1 & 1 \\
\hline 6 & 3 & 3 & 1 & 1 & 2 & 2 \\
\hline 7 & 1 & 2 & 1 & 3 & 2 & 3 \\
\hline 8 & 2 & 3 & 2 & 1 & 3 & 1 \\
\hline 9 & 3 & 1 & 3 & 2 & 1 & 2 \\
\hline 10 & 1 & 3 & 3 & 2 & 2 & 1 \\
\hline 11 & 2 & 1 & 1 & 3 & 3 & 2 \\
\hline 12 & 3 & 2 & 2 & 1 & 1 & 3 \\
\hline 13 & 1 & 2 & 3 & 1 & 3 & \\
\hline 14 & 2 & 3 & 1 & 2 & 1 & 3 \\
\hline 15 & 3 & 1 & 2 & 3 & 2 & 1 \\
\hline 16 & 1 & 3 & 2 & 3 & 1 & 2 \\
\hline & 2 & 1 & 3 & 1 & 2 & 3 \\
\hline 18 & 3 & 2 & 1 & 2 & 3 & 1 \\
\hline
\end{tabular}

Table 2

Specifications for the response variables specified by Chang (2006)

\begin{tabular}{cccccc}
\hline \multirow{2}{*}{ Signal levels } & & \multicolumn{4}{c}{ Responses } \\
& $Y_{l}$ (DLTB) & $Y_{2}$ (DNTB) & $Y_{3}$ (DSTB) \\
\cline { 2 - 6 } & LSL & LSL & Target & USL & USL \\
\hline$M_{1}$ & 55 & 7 & 10 & 13 & 6 \\
$M_{2}$ & 110 & 14 & 20 & 26 & 9 \\
$M_{3}$ & 165 & 21 & 30 & 39 & 9 \\
\hline
\end{tabular}

Table 3

Experimental Data of Chang (2006)

\begin{tabular}{|c|c|c|c|c|c|c|c|c|c|c|c|c|c|c|c|c|c|c|c|}
\hline \multirow{5}{*}{$\begin{array}{c}\begin{array}{c}\text { Trial } \\
\text { no. }\end{array} \\
1\end{array}$} & \multirow{4}{*}{$\begin{array}{c}\begin{array}{c}\text { Noise } \\
\text { factor }\end{array} \\
N_{1}\end{array}$} & \multicolumn{18}{|c|}{ Responses } \\
\hline & & \multicolumn{6}{|c|}{$\mathrm{Y}_{1}$} & \multicolumn{6}{|c|}{$\mathrm{Y}_{2}$} & \multicolumn{6}{|c|}{$\mathrm{Y}_{3}$} \\
\hline & & \multicolumn{2}{|c|}{$\mathrm{M}_{1}=10$} & \multicolumn{2}{|c|}{$\mathrm{M}_{2}=20$} & \multicolumn{2}{|c|}{$\mathrm{M}_{3}=30$} & \multicolumn{2}{|c|}{$\mathrm{M}_{1}=10$} & \multicolumn{2}{|c|}{$\mathrm{M}_{2}=20$} & \multicolumn{2}{|c|}{$\mathrm{M}_{3}=30$} & \multicolumn{2}{|c|}{$\mathrm{M}_{1}=10$} & \multicolumn{2}{|c|}{$\mathrm{M}_{2}=20$} & \multicolumn{2}{|c|}{$\mathrm{M}_{3}=30$} \\
\hline & & 61.6 & 78.2 & 128.0 & 106.0 & 230.6 & 226.9 & 7.4 & 7.2 & 16.7 & 13.2 & 23.7 & 24.1 & 1.9 & 1.9 & 4.6 & 3.7 & 7.6 & 4.6 \\
\hline & $\mathrm{N}_{2}$ & 70.8 & 57.1 & 137.3 & 160.3 & 282.2 & 252.5 & 9.1 & 10.2 & 22.8 & 17.8 & 26.2 & 26.7 & 2.0 & 2.1 & 3.9 & 4.8 & 4.7 & 4.3 \\
\hline \multirow{2}{*}{2} & $\mathrm{~N}_{1}$ & 88.3 & 93.6 & 175.2 & 181.5 & 259.7 & 304.5 & 10.1 & 8.8 & 23.4 & 22.6 & 29.6 & 30.3 & 1.8 & 2.0 & 4.0 & 2.8 & 6.0 & 3.3 \\
\hline & $\mathrm{N}_{2}$ & 72.9 & 72.7 & 174.0 & 145.5 & 258.4 & 214.6 & 8.7 & 9.2 & 19.1 & 24.1 & 31.4 & 30.5 & 1.9 & 2.2 & 3.8 & 3.5 & 6.7 & 6.1 \\
\hline \multirow{2}{*}{3} & $\mathrm{~N}_{1}$ & 80.8 & 81.1 & 154.3 & 157.4 & 238.1 & 237.8 & 10.8 & 10.5 & 20.1 & 20.9 & 30.6 & 32.4 & 1.0 & 3.2 & 4.4 & 5.4 & 8.1 & 6.7 \\
\hline & $\mathrm{N}_{2}$ & 77.2 & 83.3 & 167.1 & 159.0 & 251.8 & 257.9 & 10.6 & 10.9 & 21.8 & 23.7 & 30.5 & 32.7 & 1.4 & 2.6 & 3.8 & 3.9 & 8.0 & 2.7 \\
\hline \multirow{2}{*}{4} & $\mathrm{~N}_{1}$ & 65.9 & 71.3 & 179.2 & 151.5 & 196.1 & 221.6 & 7.6 & 7.2 & 15.3 & 14.8 & 22.5 & 22.2 & 1.7 & 2.2 & 4.1 & 4.9 & 5.9 & 6.0 \\
\hline & $\mathrm{N}_{2}$ & 83.7 & 78.4 & 135.6 & 177.0 & 246.9 & 291.8 & 8.1 & 7.6 & 14.1 & 14.7 & 21.9 & 21.8 & 2.1 & 2.6 & 3.6 & 3.3 & 7.7 & 6.9 \\
\hline \multirow{2}{*}{5} & $\mathrm{~N}_{1}$ & 79.4 & 88.6 & 121.9 & 151.6 & 248.8 & 245.1 & 11.9 & 12.7 & 25.6 & 25.7 & 36.6 & 35.7 & 2.0 & 2.1 & 2.7 & 3.8 & 4.8 & 5.8 \\
\hline & $\mathrm{N}_{2}$ & 67.8 & 87.3 & 113.6 & 141.3 & 171.5 & 244.7 & 10.5 & 11.8 & 25.8 & 26.2 & 39.1 & 33.1 & 2.6 & 1.7 & 4.1 & 3.6 & 5.5 & 5.2 \\
\hline \multirow{2}{*}{6} & $\mathrm{~N}_{1}$ & 90.5 & 87.0 & 161.8 & 169.4 & 286.9 & 236.5 & 10.2 & 10.2 & 23.7 & 21.8 & 32.0 & 32.8 & 1.8 & 2.2 & 2.5 & 4.1 & 4.9 & 5.4 \\
\hline & $\mathrm{N}_{2}$ & 87.6 & 87.8 & 160.7 & 163.9 & 231.4 & 288.7 & 10.7 & 11.2 & 22.5 & 20.0 & 34.2 & 28.0 & 1.2 & 2.0 & 4.0 & 4.4 & 6.6 & 3.1 \\
\hline & $\mathrm{N}_{1}$ & 80.9 & 74.7 & 165.9 & 163.4 & 232.2 & 246.4 & 11.7 & 12.1 & 23.3 & 23.3 & 33.5 & 32.7 & 1.9 & 1.6 & 5.2 & 5.1 & 6.6 & 5.3 \\
\hline 7 & $\mathrm{~N}_{2}$ & 69.9 & 78.7 & 141.7 & 159.1 & 260.4 & 239.7 & 11.6 & 11.6 & 22.0 & 22.5 & 33.8 & 34.0 & 1.9 & 1.7 & 3.6 & 5.1 & 5.8 & 5.1 \\
\hline 8 & $\mathrm{~N}_{1}$ & 92.3 & 71.7 & 185.7 & 154.3 & 233.1 & 240.5 & 8.3 & 8.4 & 16.9 & 18.5 & 28.1 & 27.3 & 2.8 & 1.5 & 3.4 & 3.4 & 6.2 & 3.7 \\
\hline 8 & $\mathrm{~N}_{2}$ & 104.8 & 89.4 & 216.1 & 173.0 & 340.6 & 308.9 & 8.2 & 6.1 & 18.5 & 15.1 & 29.1 & 21.1 & 1.8 & 2.2 & 4.1 & 4.3 & 9.0 & 5.0 \\
\hline 0 & $\mathrm{~N}_{1}$ & 92.8 & 59.8 & 130.7 & 142.2 & 257.3 & 266.0 & 8.6 & 9.7 & 17.3 & 18.4 & 30.9 & 34.5 & 2.3 & 1.7 & 4.5 & 5.1 & 7.4 & 6.5 \\
\hline 9 & $\mathrm{~N}_{2}$ & 82.1 & 87.0 & 175.1 & 138.2 & 161.4 & 274.4 & 8.2 & 8.4 & 18.6 & 18.0 & 31.3 & 30.9 & 2.2 & 1.2 & 4.0 & 4.0 & 7.2 & 6.7 \\
\hline 10 & $\mathrm{~N}_{1}$ & 86.0 & 100.0 & 179.1 & 175.4 & 246.9 & 244.9 & 6.9 & 6.6 & 15.8 & 16.4 & 22.2 & 24.9 & 1.7 & 1.7 & 3.9 & 5.8 & 5.2 & 8.4 \\
\hline 10 & $\mathrm{~N}_{2}$ & 81.9 & 91.2 & 190.8 & 137.8 & 293.5 & 227.1 & 8.3 & 7.2 & 14.3 & 16.6 & 23.1 & 24.2 & 2.1 & 1.8 & 3.9 & 4.8 & 4.5 & 8.0 \\
\hline & $\mathrm{N}_{1}$ & 76.3 & 78.2 & 140.0 & 154.7 & 264.1 & 260.1 & 9.5 & 9.2 & 27.9 & 25.4 & 32.3 & 33.8 & 1.0 & 1.7 & 4.1 & 4.0 & 3.9 & 5.6 \\
\hline 11 & $\mathrm{~N}_{2}$ & 67.1 & 76.0 & 169.5 & 175.4 & 239.2 & 251.8 & 10.8 & 12.2 & 23.6 & 20.4 & 26.8 & 29.2 & 2.1 & 2.3 & 5.0 & 4.6 & 6.7 & 4.5 \\
\hline 12 & $\mathrm{~N}_{1}$ & 91.4 & 81.8 & 160.1 & 167.2 & 238.5 & 197.2 & 11.8 & 10.5 & 22.9 & 25.9 & 36.9 & 36.8 & 2.1 & 1.9 & 4.0 & 3.7 & 7.4 & 6.2 \\
\hline 12 & $\mathrm{~N}_{2}$ & 85.1 & 63.8 & 123.8 & 166.4 & 233.5 & 242.0 & 11.6 & 11.5 & 22.1 & 22.6 & 32.5 & 31.9 & 1.6 & 0.9 & 4.2 & 4.1 & 2.1 & 6.9 \\
\hline & $\mathrm{N}_{1}$ & 87.9 & 82.5 & 146.5 & 167.0 & 212.4 & 222.2 & 10.1 & 10.3 & 19.9 & 22.2 & 27.1 & 27.7 & 2.0 & 2.3 & 5.0 & 4.7 & 6.7 & 7.3 \\
\hline 13 & $\mathrm{~N}_{2}$ & 57.4 & 78.5 & 91.6 & 182.3 & 250.3 & 207.3 & 10.6 & 10.1 & 20.1 & 19.2 & 27.8 & 24.6 & 2.2 & 2.1 & 4.5 & 4.7 & 7.4 & 6.2 \\
\hline 14 & $\mathrm{~N}_{1}$ & 88.1 & 78.1 & 156.7 & 170.1 & 239.1 & 215.2 & 12.1 & 10.0 & 24.1 & 20.2 & 28.0 & 35.6 & 1.7 & 2.1 & 3.8 & 3.3 & 7.2 & 4.9 \\
\hline 14 & $\mathrm{~N}_{2}$ & 81.7 & 75.7 & 140.2 & 127.8 & 241.4 & 211.7 & 11.3 & 11.8 & 23.3 & 24.4 & 32.1 & 39.9 & 1.8 & 2.7 & 3.1 & 3.8 & 4.9 & 4.7 \\
\hline 15 & $\mathrm{~N}_{1}$ & 101.8 & 78.2 & 168.3 & 180.8 & 240.6 & 235.2 & 10.2 & 7.6 & 14.9 & 19.4 & 26.9 & 19.5 & 1.7 & 2.2 & 5.3 & 3.6 & 5.0 & 7.5 \\
\hline 15 & $\mathrm{~N}_{2}$ & 80.4 & 76.5 & 206.7 & 222.6 & 325.1 & 285.2 & 8.7 & 7.2 & 16.8 & 14.1 & 26.6 & 25.1 & 2.2 & 1.9 & 4.8 & 2.6 & 3.8 & 5.5 \\
\hline & $\mathrm{N}_{1}$ & 77.4 & 75.4 & 171.7 & 159.0 & 201.3 & 219.7 & 10.4 & 10.6 & 20.7 & 22.2 & 34.4 & 30.0 & 1.9 & 2.3 & 3.1 & 4.2 & 5.8 & 3.3 \\
\hline 16 & $\mathrm{~N}_{2}$ & 72.0 & 69.5 & 189.1 & 168.6 & 254.3 & 237.3 & 11.1 & 10.9 & 20.6 & 21.4 & 30.5 & 31.4 & 2.1 & 1.6 & 4.1 & 4.8 & 5.4 & 6.9 \\
\hline 17 & $\mathrm{~N}_{1}$ & 71.4 & 69.2 & 145.0 & 152.5 & 223.8 & 218.7 & 8.8 & 8.4 & 19.0 & 13.8 & 26.2 & 24.1 & 1.5 & 2.1 & 3.8 & 4.4 & 6.9 & 4.2 \\
\hline 17 & $\mathrm{~N}_{2}$ & 77.0 & 70.5 & 158.4 & 154.0 & 218.4 & 224.1 & 9.2 & 9.0 & 16.7 & 17.4 & 27.1 & 26.2 & 1.6 & 1.8 & 3.7 & 4.4 & 4.6 & 4.2 \\
\hline & $\mathrm{N}_{1}$ & 82.8 & 67.8 & 183.7 & 175.5 & 276.1 & 254.4 & 10.7 & 9.2 & 19.8 & 20.1 & 27.3 & 31.0 & 2.5 & 2.2 & 3.1 & 3.4 & 7.7 & 7.4 \\
\hline & $\mathrm{N}_{2}$ & 85.2 & 92.0 & 154.4 & 157.6 & 249.3 & 286.1 & 11.3 & 7.7 & 19.3 & 22.6 & 29.4 & 24.6 & 1.6 & 1.8 & 3.9 & 4.7 & 6.0 & 7.3 \\
\hline
\end{tabular}


Chang and Chen (2011) proposed a neuro-genetic approach for optimizing a multi-response dynamic system and they illustrated the effectiveness of their proposed approach using a simulated example of a dynamic system containing multiple responses. This simulated example data involves three dynamic response variables named $Y_{1}$ (DLTB type), $Y_{2}$ (DNTB type) and $Y_{3}$ (DSTB type). In this study, six control factors $\mathrm{A}-\mathrm{F}$, each at three levels, were considered in an $L_{18}$ orthogonal array design. Further, a signal factor with three levels $M_{1}, M_{2}$ and $M_{3}$, and a noise factor with two levels $N_{1}$ and $N_{2}$ were considered. The experimental design used by Chang and Chen (2011) is the same as given in Table 1. The specification limits for the response variables and the simulated experimental data are available in Chang and Chen (2011). The experimental data of Chang and Chen (2011) are analysed as case study 2.

\subsection{Analysis and results}

\subsubsection{Case study 1}

It may be noted from Table 2 that Chang (2006) specified the target value of $Y_{2}$ only. However, for computation of PMSE value of a response variable, it is necessary to know its target values at all signal levels $(j=1,2, \ldots, s)$. Therefore, the target values $Y_{1}$ and $Y_{3}$ at all the signal levels are assumed as per the procedure described in section 6.1. The $\beta$ values for $Y_{1}$ and $Y_{3}$ are computed first from the experimental observations at signal level $M_{1}$. It was found that the largest $\beta$ value for $Y_{1}$ and the smallest $\beta$ value for $Y_{3}$ among all the experimental runs are 86 and 1.735 respectively. So the target values for $Y_{1}$ at the signal levels $M_{1}, M_{2}$ and $M_{3}$ are chosen as $86,2 \times 86$ and $3 \times 86$ respectively, and the target values for $Y_{3}$ at the signal levels $M_{1}, M_{2}$ and $M_{3}$ are chosen as $1.735,2 \times 1.735$ and $3 \times 1.735$ respectively. Table 4 shows the target values and specification limits for the three response variables.

\section{Table 4}

Target values and specification limits for the response variables (case study 1)

\begin{tabular}{cccccccc}
\hline \multirow{3}{*}{ Signal levels } & \multicolumn{9}{c}{ Responses } \\
\cline { 2 - 7 } & LSL & Target & LSL & Target & USL & Target & USL \\
\cline { 2 - 8 } & 55 & 86 & 7 & 10 & 13 & 1.735 & 3 \\
\hline$M_{1}$ & 110 & 172 & 14 & 20 & 26 & 3.470 & 6 \\
$M_{2}$ & 165 & 258 & 21 & 30 & 39 & 5.205 & 9 \\
\hline
\end{tabular}

The first requirement for application of the proposed optimization method is to establish the most appropriate multiple regression equations for prediction of the response variables (see step 2 of section 5). Variability at the higher signal level is expected to be higher and therefore, it is decided to establish the multiple regression equations considering $\log$ transformed values of the responses as the dependent variables. Logarithm transformation of each response variable is made and then the response model is developed for each response variable as a function of controllable variables $(\mathrm{A}-\mathrm{F})$, signal variable $(M)$ and noise variable $(N)$ using MINITAB software. The developed response models for the three response variables are given below:

$$
\begin{array}{rl}
\log _{10}\left(Y_{1}\right)=1 & .1489+0.01338 A+0.0450 B+0.1216 C+0.0042 D+0.0958 E+0.0021 F+0.5084 M \\
& -0.0562 M^{2}+0.0253 N-0.0243 C^{2}-0.0249 E^{2}-0.0098 M B-0.0097 M C-0.008 N B \\
& -0.0068 N C+0.0126 N E-0.01 N F \\
& {\left[R^{2}=0.943 \text { and adjusted } R^{2}=0.938\right]} \\
\log _{10}\left(Y_{2}\right)= & 0.2784+0.1258 A+0.2846 B-0.1459 C-0.1315 D-0.1074 E+0.1531 F+0.5486 M \\
& -0.0771 M^{2}+0.0273 N-0.0266 A^{2}-0.0651 B^{2}+0.0324 C^{2}+0.0388 D^{2}+0.0259 E^{2} \\
& -0.0323 F^{2}-0.0137 N E \\
& {\left[R^{2}=0.967 \text { and adjusted } R^{2}=0.965\right]}
\end{array}
$$




$$
\begin{aligned}
\log _{10}\left(Y_{3}\right)= & -0.2005-0.0729 A-0.0038 B+0.0191 C+0.0626 D-0.1014 E+0.0304 F+0.5667 M \\
& -0.0905 M^{2}+0.0689 N+0.0274 A^{2}-0.0207 D^{2}+0.0176 E^{2}+0.0077 M C+0.0085 M E \\
& -0.03334 N A-0.0154 N C+0.0152 N D+0.0194 N E-0.0242 N F \\
& {\left[R^{2}=0.822 \text { and adjusted } R^{2}=0.804\right] }
\end{aligned}
$$

Using ANOVA and $F$-test for significance of regression, the adequacy of the models is checked. Residual analysis in terms of normality plot of residuals, plot of residual versus predicted values and plot of residual versus individual regression variable are carried out and found satisfactory. According to step 3 in section 5, an arbitrary setting combination of control factors levels $\mathbf{x}_{k}=\mathrm{A}_{2} \mathrm{~B}_{2} \mathrm{C}_{2} \mathrm{D}_{2} \mathrm{E}_{2} \mathrm{~F}_{2}$ is selected. For the setting combination $\mathbf{x}_{k}$, the predicted values of $Y_{1}$ at all signal $\times$ noise levels, i.e. $\hat{y}_{11 k 1}, \hat{y}_{11 k 2}, \hat{y}_{12 k 1}$, $\hat{y}_{12 k 2}, \hat{y}_{13 k 1}, \hat{y}_{13 k 2}$ are obtained using Eq. (20). According to step 4 in section 5, the slope and variance around the slope for the response variable $Y_{1}$ under the setting combination $\mathbf{x}_{k}$, i.e. $\hat{\beta}_{1 k}$ and $\hat{\sigma}_{1 k}^{2}$ are computed using Eqs. (15-16), respectively. Then the MRSN for the response variable $Y_{1}$ under the setting combination $x_{k}$, i.e. $\mathrm{MRSN}_{1 k}$ is obtained using Eq. (17). Similarly, $\mathrm{MRSN}_{2 k}$ and $\mathrm{MRSN}_{3 k}$ for the response variables $Y_{2}$ and $Y_{3}$ respectively are obtained. Then, according to step 5 in section 5, the MRWSN under the setting combination $\mathbf{x}_{k}$, i.e. $\mathrm{MRWSN}_{k}$ is computed using Eq. (18). As the relative importance of the three response variables is unknown, equal weights are given to all three response variables while MRWSN $_{k}$ value is computed. All these computations are carried out in Excel worksheet.

Now according to step-6 in section 5, the 'Solver' tool of Microsoft Excel is applied to maximize the MRWSN $_{k}$ value. If integer restrictions (IR) are specified for the level values of input controllable variables, the 'Solver' tool will determine the optimal combination among the actual experimental design settings and if IR are not specified for the level values of input variables, the 'Solver' tool will determine an optimal solution over the entire experimental region of the input variables. It is decided to determine the optimal solutions under both the conditions, i.e. under IR and under no IR for the level values of the input variables. While optimizing, in both the cases, an additional constraint (absolute difference between predicted slope and target slope is less than 0.10) is added to keep the slope of the DNTB variable $\left(Y_{2}\right)$ closer to its target slope value 10. In the first case where IR are specified for the level values of input variables, the optimal solution is found to be $\mathrm{A}_{2} \mathrm{~B}_{1} \mathrm{C}_{3} \mathrm{D}_{3} \mathrm{E}_{2} \mathrm{~F}_{2}$ and in the second case where IR are not specified for the level values of input variables, the optimal solution is found to be as follows: $\mathrm{A}=1.93$, $\mathrm{B}=2.55, \mathrm{C}=2.17, \mathrm{D}=1.0, \mathrm{E}=2.06$ and $\mathrm{F}=1.68$. The expected values of each response variable at all signal $\times$ noise levels under these two optimal conditions are computed using the relevant fitted response models and then, the slope $(\hat{\beta})$, variance around slope $\left(\hat{\sigma}_{\beta}^{2}\right)$, MRSN and PMSE values for each response variable are estimated using Eqs. (14-16) and Eq. (19), respectively.

With the aim to compare the optimization performance of the proposed MRWSN-based approach with the CDF-based approach, the same experimental data are analysed again considering the OPI (which is essentially a CDF) as the objective function for the optimization. $Y_{1}, Y_{2}$ and $Y_{3}$ are DLTB, DNTB and DSTB type response variables respectively. The predicted values of the three variables at the chosen arbitrary setting are converted into appropriate double-exponential desirability functions first using in Eqs. (9-11) respectively. Then, the OPI value at the arbitrary setting is obtained using Eq. (12). The 'Solver' tool of Microsoft Excel is applied again to maximize the OPI value under both the conditions, i.e. with IR and without IR for the level values of the input variables. The optimal solution is found to be $\mathrm{A}_{3} \mathrm{~B}_{2} \mathrm{C}_{2} \mathrm{D}_{3} \mathrm{E}_{2} \mathrm{~F}_{1}$ when IR for the level values of the controllable variables are specified, and the optimal solution is found to be $\mathrm{A}=2.56, \mathrm{~B}=3.0, \mathrm{C}=1.64, \mathrm{D}=3.0, \mathrm{E}=2.02$ and $\mathrm{F}=1.20$ when no IR is specified. The expected values of each response variable at all signal $\times$ noise levels under these two optimal conditions are computed and then the expected SN ratio and PMSE for each response variable are obtained using Eq. (16) and Eq. (19), respectively. Table 5 displays the expected values of the two utility 
measures, i.e. TSN and PMSE of the individual response variables at different optimal conditions obtained by the proposed MRWSN-based approach and the CDF-based approach.

It can be noted from Table 5 that the derived optimal solutions using MRWSN-based approach result in higher TSN values in both the cases (i.e. under IR and under no IR for the level values of the control factors) than the optimal solutions derived by the CDF-based approach. This implies that the proposed MRWSN-based approach leads to better optimal solution than the CDF-based approach with respect to Taguchi's philosophy. Table 5 further reveals that the PMSE value for $Y_{1}$ at MRWSN-based optimal solutions is lesser than the PMSE value for $Y_{1}$ at the CDF-based optimal solution. The PMSE values for $Y_{2}$ and $Y_{3}$ at MRWSN-based optimal solutions is lesser or equal to the PMSE values for $Y_{2}$ and $Y_{3}$ at the CDF-based optimal solutions. Therefore, it may be concluded that MRWSN-based approach results in better optimal solution in proper statistical sense also.

Table 5

Expected TSN and PMSE values under different optimal conditions (case study 1)

\begin{tabular}{|c|c|c|c|c|c|c|c|c|}
\hline \multirow[b]{2}{*}{ Optimization Method } & \multirow[b]{2}{*}{ Optimal solution } & \multicolumn{3}{|c|}{$\mathrm{SN}$ ratio (in $\mathrm{dB}$ ) } & \multirow[b]{2}{*}{ TSN } & \multicolumn{3}{|c|}{ PMSE } \\
\hline & & $Y_{1}$ & $Y_{2}$ & $Y_{3}$ & & $Y_{1}$ & $Y_{2}$ & $Y_{3}$ \\
\hline $\begin{array}{l}\text { MRWSN-based Approach } \\
\text { (under IR for input variables) }\end{array}$ & $\mathrm{A}_{2} \mathrm{~B}_{1} \mathrm{C}_{3} \mathrm{D}_{3} \mathrm{E}_{2} \mathrm{~F}_{2}$ & 29.71 & 29.55 & 23.95 & 83.21 & 207.92 & 0.13 & 0.08 \\
\hline $\begin{array}{l}\text { CDF-based Approach } \\
\text { (under IR for input variables) }\end{array}$ & $\mathrm{A}_{3} \mathrm{~B}_{2} \mathrm{C}_{2} \mathrm{D}_{3} \mathrm{E}_{2} \mathrm{~F}_{1}$ & 27.22 & 29.55 & 22.72 & 79.49 & 250.58 & 0.18 & 0.09 \\
\hline $\begin{array}{l}\text { MRWSN-based optimization } \\
\text { (under no IR for input variables) }\end{array}$ & $\begin{array}{c}\mathrm{A}=1.93, \mathrm{~B}=2.55 \\
\mathrm{C}=2.17, \mathrm{D}=1.0 \\
\mathrm{E}=2.06 \text { and } \mathrm{F}=1.68\end{array}$ & 37.19 & 29.53 & 22.00 & 88.72 & 6.93 & 0.093 & 0.024 \\
\hline $\begin{array}{l}\text { CDF-based Optimization } \\
\text { (under no IR for input variables) }\end{array}$ & $\begin{array}{c}\mathrm{A}=2.56, \mathrm{~B}=3.0 \\
\mathrm{C}=1.64, \mathrm{D}=3.0 \\
\mathrm{E}=2.02 \text { and } \mathrm{F}=1.20\end{array}$ & 34.89 & 29.55 & 21.12 & 85.58 & 244.72 & 0.093 & 0.024 \\
\hline
\end{tabular}

\subsubsection{Case study 2}

Chang and Chen (2011) also did not specify the target values of $Y_{1}$ and $Y_{3}$. However, to facilitate computation of MSE values for $Y_{1}$ and $Y_{3}$ at different signal levels, necessary computations were made from the experimental data and then target values of these response variables were assumed as per the procedure described in section 6.1. Table 6 shows the target values and specification limits for the three response variables.

Table 6

Target values and specification limits for the response variables (case study 2)

\begin{tabular}{cccccccc}
\hline \multirow{2}{*}{ Signal levels } & \multicolumn{3}{c}{$Y_{l}(\mathrm{DLTB})$} & \multicolumn{3}{c}{ Responses } & \multicolumn{2}{c}{$Y_{3}$ (DSTB) } \\
\cline { 2 - 7 } & LSL & Target & LSL & Target & USL & Target & USL \\
\cline { 2 - 7 } & 4.8 & 9.2 & 0.6 & 1.0 & 1.4 & 16 & 28 \\
$M_{2}$ & 9.6 & 18.4 & 1.2 & 2.0 & 2.8 & 32 & 56 \\
$M_{3}$ & 14.4 & 27.6 & 1.8 & 3.0 & 4.2 & 48 & 84 \\
\hline
\end{tabular}

At first, the response model for each response variable is developed as a function of controllable variables $(A-F)$, signal variable $(M)$ and noise variable $(N)$ using MINITAB. The developed response models for the three response variables are given below:

$$
\begin{aligned}
\log _{10}\left(Y_{1}\right)= & 0.3012-0.0329 A-0.0032 B+0.105 C-0.002 D+0.1077 E+0.0062 F+0.4571 M \\
& -0.0504 M^{2}+0.0621 N-0.0186 C^{2}-0.0191 E^{2}-0.0147 A C+0.0235 A F+0.0067 M A \\
& -0.0124 M F-0.0123 N C+0.0134 N D-0.0121 N E-0.0235 N F \\
& {\left[R^{2}=0.957 \text { and adjusted } R^{2}=0.948\right] }
\end{aligned}
$$




$$
\begin{aligned}
\log _{10}\left(Y_{2}\right)= & -0.6249+0.1643 A+0.2717 B-0.2169 C-0.1038 D-0.1220 E+0.190 F+0.4930 M \\
& -0.0636 M^{2}-0.0016 N-0.0377 A^{2}-0.064 B^{2}+0.0488 C^{2}+0.0323 D^{2} \\
& +0.0242 E^{2}-0.0421 B F \\
& {\left[R^{2}=0.967 \text { and adjusted } R^{2}=0.962\right] } \\
\log _{10}\left(Y_{3}\right)= & 0.6642-0.1384 A+0.0836 B-0.1411 C-0.0172 D+0.0725 E+0.109 F+0.6209 M \\
& -0.068 M^{2}+0.024 N+0.0383 A^{2}+0.0359 C^{2}-0.0549 A B+0.0229 A C-0.0235 M C \\
& -0.0263 M F+0.0332 N B-0.0231 N E-0.0246 N F \\
& {\left[R^{2}=0.842 \text { and adjusted } R^{2}=0.810\right] }
\end{aligned}
$$

The adequacy of the developed models is checked using ANOVA and $F$-test for significance of regression. Residual analysis in terms of normality plot of residuals, plot of residual versus predicted values and plot of residual versus individual regression variable also are carried out and found satisfactory. An arbitrary setting combination of control factor levels $\mathbf{x}_{k}=\mathrm{A}_{2} \mathrm{~B}_{2} \mathrm{C}_{2} \mathrm{D}_{2} \mathrm{E}_{2} \mathrm{~F}_{2}$ is chosen. At this setting combination, the values of the all the three response variables are predicted using the fitted regression models for all signal $\times$ noise levels. Using these predicted values $\hat{\beta}_{1 k}, \hat{\beta}_{2 k}, \hat{\beta}_{3 k}, \hat{\sigma}_{\beta_{1 k}}^{2}, \hat{\sigma}_{\beta_{2 k}}^{2}$ and $\hat{\sigma}_{\beta_{3 k}}^{2}$ are computed using the relevant equations. Then, $\operatorname{MRSN}_{i k}(i=1,2,3)$ are computed using Eq. (16) and the performance metric $\mathrm{MRWSN}_{k}$ is computed using Eq. (17) considering equal weight for all the response variables (since relative importance of the response variable are unknown). Now the 'Solver' tool of Microsoft Excel is applied to maximize the $\mathrm{MRWSN}_{k}$ value considering two cases (IR are present and IR are not present for the level values of input variables). While optimizing, in both the cases, an additional constraint (absolute difference between predicted slope and target slope is less than $0.05)$ was added to keep the slope of the DNTB variable $\left(Y_{2}\right)$ closer to its target slope value 1.0. In the first case, the optimal setting combination is found to be $\mathrm{A}_{3} \mathrm{~B}_{3} \mathrm{C}_{1} \mathrm{D}_{2} \mathrm{E}_{2} \mathrm{~F}_{3}$ and in the second case, the optimal setting combination is found to be as follows: $\mathrm{A}=2.47, \mathrm{~B}=3.0, \mathrm{C}=1.53, \mathrm{D}=3.0, \mathrm{E}=2.43$ and $\mathrm{F}=2.73$. The expected values of each response variable at all signal $\times$ noise levels under the two optimal conditions are computed using the relevant fitted response models and then, the slope $(\hat{\beta})$, variance around slope $\left(\hat{\sigma}_{\beta}^{2}\right)$, SN ratio and PMSE values for each response variable are estimated using the relevant equations.

For the purpose of comparison of the optimization performance of the proposed MRWSN-based approach and CDF-based approach, the same experimental data are analysed again considering the OPI (which is essentially a CFD) as the objective function for the optimization. $Y_{1}, Y_{2}$ and $Y_{3}$ are DLTB, DNTB and DSTB type response variables respectively. The predicted values of these response variables at the chosen arbitrary setting are converted into appropriate double-exponential desirability functions using in Eq. (9), Eq. (10) and Eq. (11), respectively, and then, the OPI value at the arbitrary setting is obtained using Eq. (12). The 'Solver' tool of Microsoft Excel is applied again to maximize the OPI value under both the conditions, i.e. under IR and under no IR for the level values of the input controllable variables. Under the condition of IR for the level values of the input variables, the optimal solution is found to be $\mathrm{A}_{3} \mathrm{~B}_{3} \mathrm{C}_{1} \mathrm{D}_{3} \mathrm{E}_{2} \mathrm{~F}_{2}$, and under the condition of no IR for the level values of the input variables, the optimal solution is found to be: $\mathrm{A}=1.77, \mathrm{~B}=1.0, \mathrm{C}=1.95, \mathrm{D}=3.0, \mathrm{E}=1.80, \mathrm{~F}=1.46$. The expected values of each response variable at all signal $\times$ noise levels under these two optimal conditions are computed and then the expected SN ratio and PMSE for each response variable are obtained using the relevant equations. Table 7 displays the expected values of the two utility measures, i.e. TSN and PMSE of the individual response variable at different optimal conditions obtained by the proposed MRWSNbased approach and the CDF-based approach. 
Table 7

Expected TSN and PMSE values under different optimal conditions (case study 2)

\begin{tabular}{|c|c|c|c|c|c|c|c|c|}
\hline \multirow{2}{*}{ Optimization Method } & \multirow{2}{*}{ Optimal solution } & \multicolumn{3}{|c|}{$\mathrm{SN}$ ratio (in dB) } & \multirow{2}{*}{ TSN } & \multicolumn{3}{|c|}{ PMSE } \\
\hline & & $Y_{1}$ & $Y_{2}$ & $Y_{3}$ & & $Y_{1}$ & $Y_{2}$ & $Y_{3}$ \\
\hline $\begin{array}{l}\text { MRWSN-based Approach } \\
\text { (under IR for input variables) }\end{array}$ & $\mathrm{A}_{3} \mathrm{~B}_{3} \mathrm{C}_{1} \mathrm{D}_{2} \mathrm{E}_{2} \mathrm{~F}_{3}$ & 24.87 & 45.93 & 32.64 & 103.44 & 0.280 & 0.001 & 2.056 \\
\hline $\begin{array}{l}\text { CDF-based Approach } \\
\text { (under IR for input variables) }\end{array}$ & $\mathrm{A}_{3} \mathrm{~B}_{3} \mathrm{C}_{1} \mathrm{D}_{3} \mathrm{E}_{2} \mathrm{~F}_{2}$ & 21.79 & 45.93 & 17.74 & 85.46 & 0.504 & 0.004 & 3.627 \\
\hline $\begin{array}{l}\text { MRWSN-based optimization } \\
\text { (under no IR for input variables) }\end{array}$ & $\begin{array}{c}\mathrm{A}=2.47, \mathrm{~B}=3.0 \\
\mathrm{C}=1.53, \mathrm{D}=3.0 \\
\mathrm{E}=2.43 \text { and } \mathrm{F}=2.73\end{array}$ & 28.09 & 45.93 & 38.92 & 112.94 & 0.136 & 0.000 & 3.700 \\
\hline $\begin{array}{l}\text { CDF-based Optimization } \\
\text { (under no IR for input variables) }\end{array}$ & $\begin{array}{c}\mathrm{A}=1.77, \mathrm{~B}=1.0 \\
\mathrm{C}=1.95, \mathrm{D}=3.0 \\
\mathrm{E}=1.80 \text { and } \mathrm{F}=1.46\end{array}$ & 21.50 & 45.93 & 20.38 & 87.51 & 0.514 & 0.000 & 4.123 \\
\hline
\end{tabular}

Table 7 reveals that the derived optimal solutions based on MRWSN-based approach result in higher expected TSN values in both the cases (i.e. under IR and under no IR for the level values of the control factors) than the optimal solutions derived based on CDF-based approach. This implies that the proposed MRWSN-based approach leads to better optimal solution than the CDF-based approach with respect to Taguchi's philosophy. Table 7 further reveals that the PMSE values for $Y_{1}$ and $Y_{3}$ at MRWSN-based optimal solutions are lesser than the PMSE values for $Y_{1}$ and $Y_{3}$ at the CDF-based optimal solutions. The PMSE value for $Y_{2}$ at MRWSN-based optimal solution is lesser or equal to the PMSE value for $Y_{2}$ at the CDF-based optimal solution. Therefore, it may be concluded that MRWSN-based approach results in better optimal solution in proper statistical sense also. In this research, there is no scope to carry out the confirmatory trials with the optimal factor-level combinations. However, the results of analysis of the two case studies are indicative that proposed MRWSN-based approach for optimizing a multi-response dynamic system is promising because it not only overcome the limitations of the CDF-based approach but also results in better optimal solution with respect to utility measures like TSN and PMSE for the individual response variables.

\section{Conclusions}

Industries are increasingly emphasizing optimization of dynamic multi-response problems in the light of increasing complexities of modern manufacturing systems. CDF-based approach has gained popularity in recent years for optimization of multi-response dynamic systems. A major disadvantage with the desirability index is that it does not consider the variability of a response variable. Moreover, if the specification limits of a response variable are not provided, then the CDF cannot be computed. This article presents a new method, called multiple regression-based weighted signal-to-noise ratio (MRWSN) method, for optimization of a multi-response dynamic system. The proposed method not only overcome the limitations of CDF-based approach but also results in better optimization performance. In this method, at first appropriate multiple regression equations are fitted based on the experimental observations for prediction of the response variables, and then the values of the slope, variance around the slope and MRSN for different response variables are computed based on their predicted values instead of their observed values. The optimal setting of the input controllable variables is then determined by maximizing the MRWSN. Two sets of experimental data taken from the literature are analysed using the proposed method and the CDF-based approach. The results show that the proposed method is superior to the CDF-based approach with respect to TSN as well as PMSE values of the individual responses.

\section{References}

Bae, S. J., \& Tsui, K. L. (2006). Analysis of dynamic robust design experiment with explicit \& hidden noise variables. Quality Technology \& Quantitative Management, 3(1), 55-75. 
Box, G. (1988). Signal-to-noise ratios, performance criteria, and transformations. Technometrics, 30(1), $1-17$.

Chang, H. H. (2006). Dynamic multi-response experiments by backpropagation networks and desirability functions. Journal of the Chinese Institute of Industrial Engineers, 23(4), 280-288.

Chang, H. H. (2008). A data mining approach to dynamic multiple responses in Taguchi experimental design. Expert Systems with Applications, 35(3), 1095-1103.

Chang, H. H., \& Chen, Y. K. (2011). Neuro-genetic approach to optimize parameter design of dynamic multiresponse experiments. Applied Soft Computing, 11(1), 436-442.

Chen, S. P. (2003). Robust design with dynamic characteristics using stochastic sequential quadratic programming. Engineering Optimization, 35(1), 79-89.

Del Castillo, E., \& Montgomery, D. C. (1993). A nonlinear programming solution to the dual response problem. Journal of Quality Technology, 25(3), 199-204.

Derringer, G., \& Suich, R. (1980). Simultaneous optimization of several response variables. Journal of Quality Technology, 12(4), 214-219.

Gauri, S. K. (2014). Optimization of multi-response dynamic systems using principal component analysis (PCA)-based utility theory approach. International Journal of Industrial Engineering Computations, 5(1), 101-114.

Harrington, E. C. (1965). The desirability function. Industrial quality control, 21(10), 494-498.

Hsieh, K. L., Tong, L. I., Chiu, H. P., \& Yeh, H. Y. (2005). Optimization of a multiresponse problem in Taguchi's dynamic system. Computers \& Industrial Engineering, 49(4), 556-571.

Jeong, I. J., \& Kim, K. J. (2009). An interactive desirability function method to multi-response optimization. European Journal of Operational Research, 195(2), 412-426.

Joseph, V.R., \& Wu, C.F.J. (2002). Robust parameter design of multiple-target systems. Technometrics, 44(4), 338-346.

Khuri, A. I., \& Conlon, M. (1981). Simultaneous optimization of multiple responses represented by polynomial regression functions. Technometrics, 23(4), 363-375.

León, R. V., Shoemaker, A. C., \& Kacker, R. N. (1987). Performance measures independent of adjustment: an explanation and extension of Taguchi's signal-to-noise ratios. Technometrics, 29(3), 253-265.

Lesperance, M. L., \& Sung-Min, P. (2003). GLMs for the analysis of robust designs with dynamic characteristics. Journal of Quality Technology, 35(3), 253-263.

Liao, H. C. (2006). Multi-response optimization using weighted principal component. The International Journal of Advanced Manufacturing Technology, 27(7-8), 720-725.

Lin, D. K., \& Tu, W. (1995). Dual response surface optimization. Journal of Quality Technology, 27(1), 34-39.

McCaskey, S. D., \& Tsui, K. L. (1997). Analysis of dynamic robust design experiments. International Journal of Production Research, 35(6), 1561-1574.

Miller, A., \& Wu, C. F. J. (1996). Parameter design for signal-response systems: A different look at Taguchi's dynamic parameter design. Statistical Science, 11(2), 122-136.

Montgomery, D. C., Peck, E. A., \& Vining, G. G. (2015). Introduction to linear regression analysis. New York: John Wiley \& Sons.

Pal, S., \& Gauri, S. K. (2010). Multi-Response Optimization Using Multiple Regression-Based Weighted Signal-to-Noise Ratio (MRWSN). Quality Engineering, 22(4), 336-350.

Pignatiello, Jr., \& Joseph, J. (1993). Strategies for robust multi-response quality engineering. IIE Transactions, 25(3), 5-15.

Su, C. T., Chen, M. C., \& Chan, H. L. (2005). Applying neural network and scatter search to optimize parameter design with dynamic characteristics. Journal of the Operational Research Society, 56(10), 1132-1140.

Su, C. T., \& Tong, L. I. (1997). Multi-response robust design by principal component analysis. Total Quality Management, 8(6), 409-416.

Taguchi, G. (1986). Introduction to quality engineering: designing quality into products and processes. Tokyo, Japan: Asian Productivity Organization. 
Tong, L. I., Chen, C. C., \& Wang, C. H. (2007). Optimization of multi-response processes using the VIKOR method. The International Journal of Advanced Manufacturing Technology, 31(11-12), 1049-1057.

Tong, L.I., \& Hsieh, K.L. (2000). A novel means of applying artificial neural networks to optimize multiresponse problem. Quality Engineering, 13(1), 11-18.

Tong, L. I., Wang, C. H., Houng, J. Y., \& Chen, J. Y. (2001). Optimizing dynamic multi-response problems using the dual-response-surface method. Quality Engineering, 14(1), 115-125.

Tong, L. I., Wang, C. H., Chen, C. C., \& Chen, C. T. (2004). Dynamic multiple responses by ideal solution analysis. European Journal of Operational Research, 156(2), 433-444.

Tong, L. I., Wang, C. H., \& Tsai, C. W. (2008). Robust design for multiple dynamic quality characteristics using data envelopment analysis. Quality and Reliability Engineering International, 24(5), 557-571.

Tsui, K. L. (1999). Modeling and analysis of dynamic robust design experiments. IIE Transactions, 31(12), 1113-1122.

Tsui, K. (2001). Response model analysis of dynamic robust design experiments, in: Lenz, H.J., Wilrich, P.T. (Eds.) Frontiers in Statistical Quality Control 6. Physica-Verlag Heidelberg, New York, pp. 360370.

Wang, C. H., \& Tong, L. I. (2004). Optimization of dynamic multi-response problems using grey multiple attribute decision making. Quality Engineering, 17(1), 1-9.

Wang, C. H. (2007). Dynamic multi-response optimization using principal component analysis and multiple criteria evaluation of the grey relation model. The International Journal of Advanced Manufacturing Technology, 32(5-6), 617-624.

Wang, J., Ma, Y., Ouyang, L., \& Tu, Y. (2016). A new Bayesian approach to multi-response surface optimization integrating loss function with posterior probability. European Journal of Operational Research, 249(1), 231-237.

Wasserman, G. S. (1996). Parameter design with dynamic characteristics: A regression perspective. Quality and Reliability Engineering International, 12(2), 113-117.

$\mathrm{Wu}, \mathrm{C}$. F. J. (2004). Optimization of correlated multiple quality characteristics using desirability function. Quality Engineering, 17(1), 119-126.

Wu, C.F.J., \& Hamada, M. (2000). Experiments: Planning, analysis, and parameter design optimization. New York: Wiley-Interscience.

Wu, F. C., \& Yeh, C. H. (2005). Robust design of multiple dynamic quality characteristics. The International Journal of Advanced Manufacturing Technology, 25(5-6), 579-588.

Wu, F. C. (2009). Robust design of nonlinear multiple dynamic quality characteristics. Computers \& Industrial Engineering, 56(4), 1328-1332.

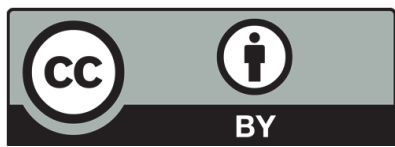

(C) 2016 by the authors; licensee Growing Science, Canada. This is an open access article distributed under the terms and conditions of the Creative Commons Attribution (CCBY) license (http://creativecommons.org/licenses/by/4.0/). 